\title{
Enfermedad de Forestier-Rotés Querol: un proceso continuo desde la Atenas de Pericles hasta el mundo de Almodóvar
}

\author{
Forestier-Rotés Querol disease: a continuous process \\ from the Athens of Pericles to the world of Almodóvar
}

Julio Montes-Santiago

Servicio de Medicna interna. Complejo Hospitalario Universitario de Vigo.

\section{RESUMEN}

Se presentan 2 casos, separados por un intervalo de más de 2500 años, de Enfermedad de Forestier - Rotés-Querol, uno de ellos procedentes de un enterramiento de la Atenas de Pericles y el otro actual para ejemplificar su presencia continua en la historia de la patología humana. Se realiza una sucinta revisión histórica sobre su separación de las espondilopatías inflamatorias anquilosantes, se revisan sus posibles manifestaciones clínicas y radiológicas y se menciona su importante papel en el desarrollo de la última película de Pedro Almodóvar, Dolor y Gloria.

Palabras clave: Enfermedad de Forestier-Rotés Querol.

\section{INTRODUCCIÓN}

En 1950 Jaume Rotés Querol, que hacía una estancia en Aix-les-Bains, Francia, junto al afamado reumatólogo Jacques Forestier, revisaba junto con otro colaborador de Forestier, François Jaqueline, la historia de 200 casos de pacientes con Espondilitis anquilosante (EA). Descubre 9 casos "rarísimos, porque todos son viejos - media de 65 años-, no tienen dolor, no tienen sacroileitis y tienen unas formaciones muy particulares"1. Ello llamó inmediatamente la atención de Forestier, con lo que descubren nuevos casos procedentes de necropsia. Estos hallazgos son comunicados y revisaron los hallazgos de autopsia, añadiendo nuevos pacientes. Estos estudios son presentados en la Reunión de la Ligue Française contre le Rhumatisme y publicados en el mismo año 1950 en Annals of the Rheumatic Diseases ${ }^{2}$. Con ello adquiría carta de naturaleza en el ámbito científico, como diferente de la EA, la "hiperostosis vertebral anquilosante senil" - enfermedad de Forestier-Rotés-Querol (EFRQ)-, aunque más tarde perderá el apelativo senil por descubrirse también en pacientes entre 40 y 50 años.

En 1975, Resnick y colbs. ${ }^{3}$ la denominan hiperostosis esquelética idiopática difusa (DISH) y fijan sus criterios radiológicos definitorios. 1) Osificación de la porción anterolateral de al menos 4 cuerpos vertebrales. 2) Preservación de la altura discal del nivel afectado. 3) Ausencia del fenómeno del vacío o de esclerosis marginal en los cuerpos vertebrales. 4) Ausencia de anquilosis de las articulaciones interapofisarias 0 de alteraciones en las sacroilíacas.

Presentamos 2 casos separados por más de 2500 años, uno que data del siglo $\mathrm{V}$ aC., y otro actual.

\begin{abstract}
We present two cases of Forestier-Rotés-Querol disease, separated by an interval of more than 2500 years, one of them coming from a burial in the Athens of Pericles and the other from the present. This exemplify its continuous presence in the history of human pathology. A brief historical review of their separation from ankylosing inflammatory spondylopathies is carried out their possible clinical and radiological manifestations are reviewed, and it is mentioned an important role in the development of the latest film by Pedro Almodóvar, Dolor y Gloria.
\end{abstract}

Key words: Forestier-Rotés Querol disease.

\section{CASOS}

Caso 1. Excavaciones realizadas en 1891 en el antiguo cementerio ateniense de Kerameikos (Fig. 1A) desvelaron un sarcófago de mármol con el esqueleto en buenas condiciones de un hombre maduro, junto con restos de parras de vino y 2 lekythoi (vasos funerarios) procedentes del taller del pintor Tymbos, según la tradición de enterramiento frecuente en la Grecia clásica. No existen más datos sobre dicho personaje, pero su inspección a traves de las vitrinas de cristal del Museo Arqueológico Nacional de Atenas muestra en columna y costillas inequívocos signos de osificación "en manto céreo" del ligamento vertebral anterior dorsal (Fig. 1B). No hay afectación sacroilíaca. Además presentaba cálculos y carencias de piezas dentales. Dicho sarcófago (T35), datado entre 460-450 aC es, pues, contemporáneo de Pericles (495-429 aC).

Caso 2. Varón de 70 años, calderero jubilado, remitido a nuestra consulta por parestesias nocturnas en miembros inferiores. Refería además dolores lumbares y cervicales que empeoraban con los cambios climáticos. El paciente era hipertenso y tenía diabetes mellitus (DM) con HbA1c actual: 6,2\%. Presentaba dolor a la motilidad cervical y percusión lumbar, con reflejos y sensibilidad conservados. El electromiograma mostró datos compatibles con polineuropatía sensitivo-motora con predominio axonal y distal, sin afectación radicular, y que fue atribuida a la DM. En Rx. y TAC se observan osteofitos dorsolumbares anterolaterales compatibles con DISH, con espacios intersomáticos conservados (Fig. 2A y fig. 2B). Había estenosis de canal L4-L5 y foraminal L5-S1. Fue tratado con AINES, pregabalina y fisioterapia.

\section{DISCUSIÓN}

La DISH o EJRQ es un proceso no inflamatorio idiopático en el que se produce osificación de las entesis y ligamentos vertebrales anterolaterales. No obstante, a diferencia de las descripciones iniciales se ha comprobado que pueden ser 
Fig. 1A: Vista del cementerio de Kerameikos en Atenas. Fig. 1B: Varón enterrado en sarcófago T35 con el característico manto "cereo" óseo (flecha), sugestivo de enfermedad de Forestier-Rotés Querol. Museo Arqueológico Nacional de Atenas (Fotografías. cortesía de Y. Carbajales).

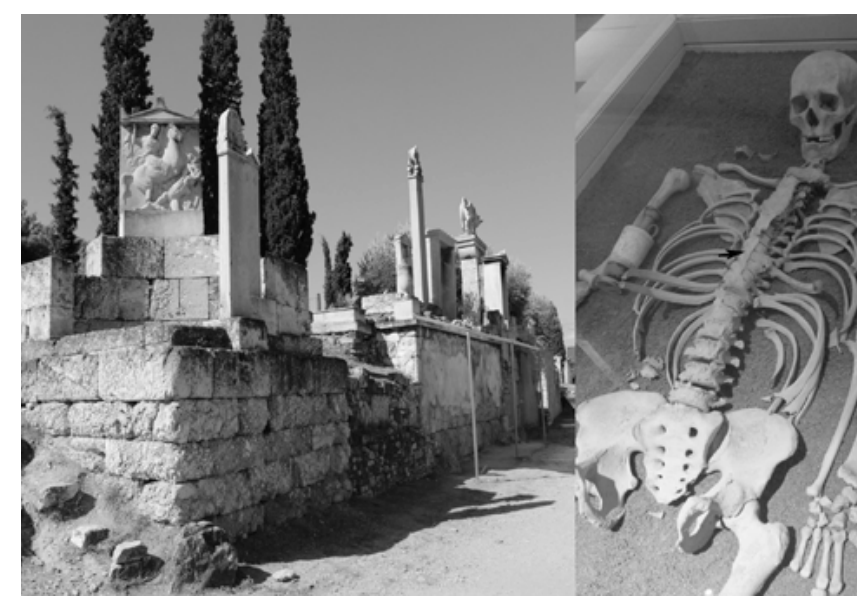

también afectados los ligamentos de articulaciones periféricas como los rotulianos, tendón de Aquiles, fascia plantar, etc 4 .

Aunque diferenciada sólo a partir de 1950 de la EA y otras espondilopatías inflamatorias, se ha documentado su existencia en investigaciones arqueológicas realizadas sobre distintas poblaciones medievales en zonas de Grecia, Inglaterra, Suiza, Francia, etc ${ }^{5,6}$.

Actualmente se considera un proceso común, afectando hasta el $10 \%$ de la población, sobre todo varones a partir de 60 años. Puede ocasionar pocos síntomas 0, por el contrario, dolor y rigidez dorso lumbares y en casos más graves las excrecencias óseas pueden comprimir mecánicamente el esófago produciendo síndromes obstructivos ${ }^{7}$. Se ha relacionado con factores mecánicos, exceso de flúor en el agua 0 de vitamina $A$, uso prolongado de isotretinoína y hábitos nutricionales y en asociación frecuente con síndrome metabólico y diabetes.

Desde el punto de vista fisiopatológico, se supone que todos estos procesos - mecánicos, metabólicos, genéticos, de inmovilidad, ambientales o dietéticos, etc - podrían estimular varios factores de crecimiento como el IGF1, 0 el NFkappa B y otros, que activarían la actividad osteoblástica y ocasionarían la proliferación ósea4. Su tratamiento es conservador con antiinflamatorios, fisioterapia, pero los casos graves de disfagia pueden requerir resección de los segmentos hiperostóticos ${ }^{8}$. En su última película, Dolor y Gloria, (n $\left.{ }^{0} 21\right)$, probablemente la más personal de Pedro Almodóvar, el personaje de Antonio Banderas - que representa un director de cine inmerso en una esterilidad creativa agobiante - sufre quejas mal caracterizadas, entre ellas disfagia y algún aparatoso episodio de broncoaspiración ${ }^{9}$. Sus médicos creen que es cáncer, pero cercano el final le revelan que sus síntomas se deben a la "Enfermedad de Forestier", le muestran en la radiografía
Fig. 2 A: Rx. de columna lumbar. Fig. 2B: TAC dorso-lumbar con las hiperostosis del ligamento vertebral anterior.

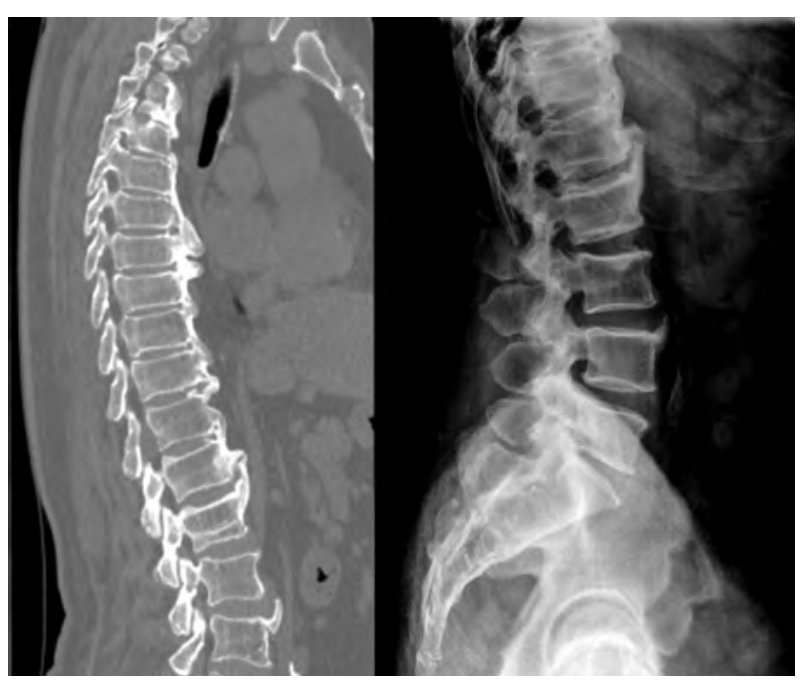

las características hiperostosis dorsales que comprimen su esófago y le proponen una intervención...

Aunque solo reconocida como entidad separada de la EA en 1950, los dos casos aportados sirven para ilustrar la continuidad histórica de este proceso, que ha merecido la atención de uno de nuestros más universales cineastas.

Derecho a la privacidad y consentimiento informado. El autor ha obtenido consentimiento informado del paciente $n^{\circ} 2$ referido en el artículo. Dicho paciente autorizó que las imágenes de su proceso pudieran ser utilizadas con fines científicos.

\section{BIBLIOGRAFÍA}

1. Jaume Rotés i Querol. La malaltia de Forestier-Rotés-Querol. En. Guardiola E, Baños JE. Eponímia médica catalana (II). Quaderns de la Fundació Dr. Antoni Esteve. 2011, 22: 135-9.

2. Forestier J, Rotes-Querol J. Senile ankylosing hyperostosis of the spine. Ann Rheum Dis. 1950; 9:321-30.

3. Resnick D, Shaul SR, Robins JM. Diffuse idiopathic skeletal hyperostosis (DISH): Forestier's disease with extraspinal manifestations. Radiology. 1975: 115: 513-24.

4. Sarzi-Puttini P, Fabiola Atzeni F. New developments in our understanding of DISH (diffuse idiopathic skeletal hyperostosis). Curr Opin Rheumatol. 2004; 16:287-92.

5. Crubezi E. Diffuse idiopathic skeletal hyperostosis. Diagnosis and importance in paleopathology. J Paleopathol. 1990; 2: 107-18.

6. Tritsaroli P. Skeletal evidence of Diffuse Idiopathic Skeletal Hyperostosis (DISH) in a collective burial from Byzantine Greece Anthropol Rev 2018; 81: 53-65.

7. Nascimento FA, Maranha Gatto LA, Oliver Lages R, Mello Neto H, Demartini Jr. Z, Koppe GL. Diffuse idiopathic skeletal hyperostosis: A review. Surg Neurol Int. 2014; 5(Suppl. 3): S122-S125

8. Silva I, Carvalho R. Doença de Forestier-Rotes-Querol - quando afeta ao dia-a-dia. Galicia Clin 2018; 79: 101

9. Iriberri A. Así es la extraña enfermedad de Antonio Banderas en la última película de Almodóvar.25/03/2019.Disponible_en:https://www.elespanol.com/ciencia/ salud/20190325/extrana-enfermedad-antonio-banderas-ultima-pelicula-almodovar/385961747_0.html. [consultado, 25/04/2019]. 